\title{
A NON-POWER SERIES APPROACH TO WIMAN-VALIRON TYPE THEOREMS
}

\author{
P. C. Fenton and John Rossi* \\ University of Otago, Department of Mathematics and Statistics \\ P. O. Box 56, Dunedin, New Zealand; pfenton@maths.otago.ac.nz \\ Virginia Tech, Department of Mathematics \\ Blacksburg, VA 24061-0123, U.S.A.; rossij@math.vt.edu
}

\begin{abstract}
Using ideas developed by Bergweiller, Rippon and Stallard, we improve three theorems of Wiman-Valiron type without the use of power series.
\end{abstract}

\section{Introduction}

An important consequence of the Wiman-Valiron theory for an entire function $f$ is a precise estimate of that function at points $z$ sufficiently close to points $z_{r}$ at which the maximum modulus of $f$ is attained on circles of radius $r$. The estimate takes the form

$$
f(z) \sim f\left(z_{r}\right)\left(\frac{z}{z_{r}}\right)^{N(r, f)}
$$

where $N(r, f)$ is the so-called central index of $f$.

These estimates have had applications in the theory of ordinary differential equations with entire coefficients as well as in the iteration of entire functions. Similar estimates were obtained by the authors for functions analytic in the unit disc [6], for entire functions of several variables $[4,7]$ and for classes of subharmonic functions which are well approximated by entire functions in large discs [8]. Generally such results depend crucially on an analysis of the coefficients of the power series representation of $f$ (see for example [9]) and as such the methods are not generalizable to functions with no such representation.

Using only certain convexity properties of the maximum of subharmonic functions and standard harmonic measure estimates, Bergweiller, Rippon and Stallard [1, 2] were able to extend (1.1) to certain classes of meromorphic functions. Modifying their methods, Langley and Rossi [11] obtained similar results for an analagous class of meromorphic functions in the unit disc.

In this note we show that the methods in [2] and [1] can also be modified to give a non-power series proof and improvement to the main result in [8] as well as a more general version of the main result in [4]. Finally by assuming that $f$ has finite order we improve a general result of Bergweiller on the size of the set where (1.1) holds.

doi:10.5186/aasfm.2016.4124

2010 Mathematics Subject Classification: Primary 30D30; Secondary 30D20, 32A15.

Key words: Wiman-Valiron theory, subharmonic function, direct track.

*The author acknowledges generous support from the University of Otago during a visit in February 2015. 


\section{Statement of Theorem 1}

We first extend the result in [8]. Suppose that $u$ is subharmonic in the plane with $B(r, u)=\max _{|z|=r} u(z)$ and

$$
a(r, u)=\frac{d B(r, u)}{d \log r} .
$$

Since $B$ is a convex function of $\log r$, we have that $a$ is increasing with $r$.

We use $\Delta(z, r)$ and $C(z, r)$ to denote the open disc and the circle with radius $r$ and centre $z$. We call $\Delta(z, T)$ a Wiman-Valiron disc for a function $u$ subharmonic in the plane if $u(z)=B(|z|, u)$ and

$$
u(w)=\log |g(w)|
$$

for all $w \in \Delta(z, T)$, where $g$ is analytic and nonzero in $\Delta(z, T)$.

As in [8] we restrict our attention to functions which are harmonic near points where they are large. To be precise we define $\psi:\left[t_{0}, \infty\right) \rightarrow(0, \infty)$ to be any function satisfying

$$
\int_{t_{0}}^{\infty} \frac{d t}{\psi(t)}<\infty
$$

and

These conditions imply that for all $t \geq t_{0}$

$$
1 \leq \frac{t \psi^{\prime}(t)}{\psi(t)} \leq L<2
$$

$$
c_{1} t \leq \psi(t) \leq c_{2} t^{L}
$$

where $c_{1}, c_{2}>0$ are constants depending on $t_{0}$ and $\psi\left(t_{0}\right)$.

Let $u\left(z_{r}\right)=B(r, u)$, where $\left|z_{r}\right|=r$, and set

$$
\tau(r)=\max (r, B(r, u))
$$

and

$$
T(r)=\frac{r}{\sqrt{\psi(a(r, u))}} .
$$

We say that $u$ is admissible if

$$
a(r, u) \rightarrow \infty
$$

and there are numbers $c>1$ and $K>0$ and a set $\mathcal{E}$ of finite logarithmic measure such that whenever $r \notin \mathcal{E}$ is sufficiently large and $z \in \Delta\left(z_{r}, 4 T(r)\right)$ satisfies

$$
u(z)>B(|z|, u)-K \log \tau(|z|),
$$

then $u$ is harmonic in $\Delta\left(z, \tau(|z|)^{-c}\right)$.

We will prove:

Theorem 2.1. Let $u$ be subharmonic in $\mathbf{C}$ and admissible. Then there is a set $E \subseteq \mathbf{R}^{+}$of finite logarithmic measure such that, for all $r \in \mathbf{R}^{+} \backslash E, \Delta\left(z_{r}, T(r)\right)$ is a Wiman-Valiron disc for $u$. Further for $z \in \Delta\left(z_{r}, T(r)\right)$,

$$
g(z)=(1+o(1)) g\left(z_{r}\right)\left(\frac{z}{z_{r}}\right)^{a(r, u)}
$$

and for any positive integer $q$

$$
\frac{g^{(q)}(z)}{g(z)}=(1+o(1))\left(\frac{a(r, u)}{z}\right)^{q}
$$


uniformly as $r \rightarrow \infty$, where $g$ is given by (2.2).

Remarks. 1. We note that a general subharmonic function may not possess Wiman-Valiron discs - e.g. a function with a fairly thick set of point masses. Thus some restriction on the functions akin to admissible is a necessary one. After the proof of Theorem 2.1 we will show that the subharmonic functions considered in [2] and [1] are automatically admissible and as such this theorem can be considered a generalization of Theorem 2.2 in [2] or Theorem 1.1 in [1].

2. Theorem 1 extends and improves Theorem 1 in [8]. First of all the WimanValiron discs are centred at the points where the maximum is attained and not just near such points as was the case in the original theorem. Secondly the definition of admissibilty only applies near the maximum points $z_{r}$. More importantly, Theorem 1 removes a very unnatural lower growth hypothesis on $u$ which marred the original theorem. Finally the new proof greatly simplifies the old one.

3. For Theorem 2.1 to be meaningful, the discs in the definition of admissible must be much smaller than the Wiman-Valiron discs. This is the case since by (3.1) below and since $\psi$ increases we have that

$$
\frac{\tau(r)^{-c}}{T(r)} \rightarrow 0
$$

as $r \rightarrow \infty$ outside of a set of finite logarithmic measure.

\section{Proof of Theorem 2.1}

We need some preliminary results. Fix $\delta_{0} \in(0,1)$ such that $1+\delta_{0}<c$. Then there exists a set $E_{1}$ of finite logarithmic measure such that for $r \notin E_{1}$

$$
a(r, u) \leq B(r, u)^{1+\delta_{0}} .
$$

Indeed, if $E_{1}$ is the set on which (3.1) fails, then

$$
\int_{E_{1}} \frac{d r}{r} \leq \int_{E_{1}} \frac{a(r, u)}{B(r, u)^{1+\delta_{0}}} \frac{d r}{r} \leq \int_{0}^{\infty} \frac{a(r, u)}{B(r, u)^{1+\delta_{0}}} \frac{d r}{r}=\int_{0}^{\infty} \frac{B^{\prime}(r, u)}{B(r, u)^{1+\delta_{0}}} d r<\infty
$$

since $B(r, u) \rightarrow \infty$.

Finally by a result of Bergweiller (end of section 2 in [1]) there is a set $E_{2} \in \mathbf{R}^{+}$ of finite logarithmic measure such that for all $r \notin E_{2}$

$$
B(s, u) \leq B(r, u)+a(r, u) \log \frac{s}{r}+o(1), \quad r \rightarrow \infty
$$

for all $s$ satisfying

$$
\left|\log \frac{s}{r}\right| \leq 16 \rho(r)
$$

where

$$
\rho(r)=T(r) / r=\frac{1}{\sqrt{\psi(a(r, u))}} .
$$

By the convexity of $B$ we also obtain for $s, r>0$ that

$$
B(s, u) \geq B(r, u)+a(r, u) \log \frac{s}{r} .
$$

We now let $E=E_{1} \cup E_{2} \cup \mathcal{E}$. Thus if $r \notin E$ we have by (3.2), (3.3), (3.5) and (2.7) that for all $z, w \in \Delta\left(z_{r}, 12 T(r)\right)$,

$$
|B(|z|, u)-B(|w|, u)| \leq 24 a(r, u) \rho(r)+o(1)
$$


as $r \rightarrow \infty$. Then by (2.4) and (3.1)

$$
|B(|z|, u)-B(|w|, u)|=o(B(r, u)) .
$$

By $(2.4)$

and so we have that

$$
|| z|-| w|| \leq 24 T(r)=o(r)
$$

$$
|\tau(|z|)-\tau(|w|)|=o(\tau(r))
$$

$z, w \in \Delta\left(z_{r}, 12 T(r)\right)$.

Set

$$
u_{1}(z)=u(z)-B(r, u)-a(r, u) \log \frac{|z|}{r} .
$$

Note that $u_{1}$ is subharmonic in $\Delta\left(z_{r}, 12 T(r)\right)$ and since $u(z) \leq B(|z|, u)$ we have by (3.2) that

$$
u_{1}(z) \leq \epsilon(r)=o(1)
$$

uniformly as $r \rightarrow \infty, r \notin E$, for $z \in \Delta\left(z_{r}, 12 T(r)\right)$.

We claim that $u$ is harmonic in $\Delta\left(z_{r}, 4 T(r)\right)$. Indeed, if not, then we can find $\zeta \in$ $\Delta\left(z_{r}, 4 T(r)\right)$ at which $u$ is not harmonic. By the definition of admissible this would imply that for all sufficiently large $r$, (2.8) fails on the entire circle $C\left(\zeta, \tau(|\zeta|)^{-c} / 2\right)$. From (3.2) and (2.8) we obtain for all $z$ on this circle

$$
u(z) \leq B(|z|, u)-K \log \left(\tau(|z|) \leq u\left(z_{r}\right)+a(r, u) \log \frac{|z|}{r}+\epsilon(r)-K \log (\tau(|z|))\right.
$$

where $\epsilon(r) \rightarrow 0$ as $r \rightarrow \infty$.

Let $r_{1}=\tau(|\zeta|)^{-c} / 2, r_{2}=8 T(r)$ and denote by $h(z)$ the first three terms on the right hand side of (3.9). Then by (3.8), (3.9) and (3.7)

$$
h(z)-K \log \tau(|\zeta|) \frac{\log \left(r_{2} /|z-\zeta|\right)}{\log \left(r_{2} / r_{1}\right)}
$$

is a harmonic majorant for $u$ in the annulus $A=\left\{z: r_{1} \leq|z-\zeta| \leq r_{2}\right\}$.

We claim that $z_{r} \in A$. Indeed since $u$ is admissible, $u$ is harmonic in $\Delta\left(z_{r}, \tau(r)^{-c}\right)$. This together with (3.7) gives $\left|z_{r}-\zeta\right|>\tau(r)^{-c}>\tau(|\zeta|)^{-c} / 2$ for all large $r \notin E$ and proves the claim.

Thus we obtain

$$
\begin{aligned}
u\left(z_{r}\right) & \leq u\left(z_{r}\right)-K \log \tau(|\zeta|) \frac{\log \left(r_{2} /\left|z_{r}-\zeta\right|\right)}{\log r_{2} / r_{1}}+\epsilon(r) \\
& \leq u\left(z_{r}\right)-K \log \tau(|\zeta|) \frac{\log 2}{\log 16 T(r) \tau(|\zeta|)^{c}}+\epsilon(r) \\
& \leq u\left(z_{r}\right)-\frac{1}{2} K \log \tau(|\zeta|) \frac{\log 2}{\log \tau(|\zeta|)^{c+1}}+\epsilon(r) \\
& =u\left(z_{r}\right)-\frac{1}{2} K \frac{\log 2}{c+1}+\epsilon(r) .
\end{aligned}
$$

The last inequality occurs for large $r$ and follows from the fact that $T(r)=o(\tau(r))$ and (3.7). Thus we have the contradiction $u\left(z_{r}\right)<u\left(z_{r}\right)$.

Proceeding as in [2] we see that $u=\log |g|$ where $g$ is analytic and nonzero in $\Delta\left(z_{r}, 4 T(r)\right)$. The function

$$
g_{1}(z)=\log \left(\frac{g(z)}{g\left(z_{r}\right)}\left(\frac{z_{r}}{z}\right)^{a(u, r)}\right)
$$


is analytic in $\Delta\left(z_{r}, 4 T(r)\right)$. Here the branch is chosen so that $g_{1}\left(z_{r}\right)=0$. By (3.8) we have that for $z \in \Delta\left(z_{r}, 4 T(r)\right), \Re g_{1}(z)=u_{1}(z) \rightarrow 0$ as $r \rightarrow \infty, r \notin E$. By the Borel-Carathéodory inequality we obtain that

$$
\left|g_{1}(z)\right| \rightarrow 0
$$

for $z \in \Delta\left(z_{r}, 2 T(r)\right)$ and $(2.9)$ is proved.

To prove (2.10) we note that by $(3.11),(2.4),(2.7)$ and Cauchy's theorem we obtain for all non-negative integers $q$ and $z \in \Delta\left(z_{r}, T(r)\right)$

$$
g_{1}^{(q)}(z) \leq o\left(T(r)^{-q}\right)=o\left(\psi(a(r, u))^{q / 2} / r^{q}\right)=o(a(r, u) / r)^{q}
$$

uniformly in $r$.

Now

$$
\log g(z)=\log g\left(z_{r}\right)+a(r, u) \log \frac{z}{z_{r}}+g_{1}(z)
$$

for $z \in \Delta\left(z_{r}, 2 T(r)\right)$. So by $(3.12)$

$$
\frac{g^{\prime}(z)}{g(z)}=(1+o(1)) \frac{a(r, u)}{z}
$$

for $z \in \Delta\left(z_{r}, T(r)\right)$ and we have (2.10) for $q=1$. Further

$$
\frac{g^{\prime \prime}(z)}{g(z)}=\left(\frac{g^{\prime}(z)}{g(z)}\right)^{\prime}+\left(\frac{g^{\prime}(z)}{g(z)}\right)^{2}
$$

and so by (3.13), (3.12) and (2.7) we obtain (2.10) for $q=2$. We proceed as in [11] by induction noticing that for all $q$

$$
\frac{g^{(q+1)}(z)}{g(z)}=\left(\frac{g^{(q)}(z)}{g(z)}\right)^{\prime}+\frac{g^{(q)}(z)}{g(z)} \frac{g^{\prime}(z)}{g(z)} .
$$

Theorem 1 is proved.

In [2] and [1] the subharmonic functions $u$ have the form $u=\max (\log |g|, 0)$ in a domain $D$, a direct tract of the meromorphic function $g$ in which $\log |g|>0$. Such functions are apriori admissible. Indeed, by equation (2.5) in [2], $u$ satisfies (2.7). As in [2] we have $\Delta(z, 2 T(r)) \subseteq D$, forcing $u$ to be harmonic there. By (2.11) we see that $u$ is admissible in $\Delta(z, T(r))$.

\section{Statement of Theorem 4.1}

We denote the polydisc centred at $\left(z_{1}, z_{2}\right)$ with radii $\left(r_{1}, r_{2}\right)$ by $D\left(z_{1}, z_{2} ; r_{1}, r_{2}\right)$ Let $f\left(z_{1}, z_{2}\right)$ be an entire function in $\mathbf{C}^{2}$ and let $M\left(r_{1}, r_{2}\right)=M\left(r_{1}, r_{2}, f\right)$ be the maximum of $|f|$ on the polydisc $D\left(0,0 ; r_{1}, r_{2}\right)$. We note that $\log M$ is a convex function in the $\left(\log r_{1}, \log r_{2}\right)$ plane and increases in each variable separately. We denote

$$
a_{1}(r, u)=\frac{\partial B\left(r_{1}, r_{2}\right)}{\partial \log r_{1}}, \quad a_{2}(r, u)=\frac{\partial B\left(r_{1}, r_{2}\right)}{\partial \log r_{2}} .
$$

We define the two dimensional logarithmic measure of a set $F \subseteq(0, \infty) \times(0, \infty)$ by

$$
\operatorname{logmeas} F=\iint_{F} \frac{d r d s}{r s} .
$$

We let $\psi$ be as in Section 2 and set

$$
T_{1}\left(r_{1}, r_{2}\right)=\frac{r_{1}}{4 \sqrt{\psi\left(a_{1}\left(r_{1}, r_{2}\right)\right)}}, \quad T_{2}\left(r_{1}, r_{2}\right)=\frac{r_{2}}{4 \sqrt{\psi\left(a_{2}\left(r_{1}, r_{2}\right)\right)}} .
$$

We will prove 
Theorem 4.1. Let $f\left(z_{1}, z_{2}\right)$ be an entire function in $\mathbf{C}^{2}$ with $\left|z_{1, r_{1}}\right|=r_{1},\left|z_{2, r_{2}}\right|=$ $r_{2}$ and $\left|f\left(z_{1, r_{1}}, z_{2, r_{2}}\right)\right|=M\left(r_{1}, r_{2}\right)$. Then there is a set $E \subseteq(0, \infty) \times(0, \infty)$ and $\kappa>0$ for which

$$
\text { logmeas } E \cap\left(0, R_{1}\right) \times\left(0, R_{2}\right) \leq \kappa\left(\log R_{1}+\log R_{2}\right)
$$

for all large $R_{1}, R_{2}$ and such that for all $\left(z_{1}, z_{2}\right)$ in

$$
D=D\left(z_{1, r_{1}}, z_{2, r_{2}} ; T_{1}\left(r_{1}, r_{2}\right), T_{2}\left(r_{1}, r_{2}\right)\right)
$$

and $\left(r_{1}, r_{2}\right) \in\left(\mathbf{R}^{+}\right)^{2} \backslash E$,

$$
\frac{f\left(z_{1}, z_{2}\right)}{f\left(z_{1, r_{1}}, z_{2, r_{2}}\right)}=(1+o(1))\left(\frac{z_{1}}{z_{1, r_{1}}}\right)^{a_{1}\left(r_{1}, r_{2}\right)}\left(\frac{z_{2}}{z_{2, r_{2}}}\right)^{a_{2}\left(r_{1}, r_{2}\right)}
$$

uniformly as $r_{1} \rightarrow \infty$ and $r_{2} \rightarrow \infty$.

Remarks. 1. One would hope that (4.4) would imply that

$$
\frac{\partial^{p_{1}+p_{2}}}{\partial z_{1}^{p_{1}} \partial z_{2}^{p_{2}}} f\left(z_{1}, z_{2}\right)=(1+o(1))\left(\frac{a_{1}\left(r_{1}, r_{2}\right)}{z_{1}}\right)^{p_{1}}\left(\frac{a_{2}\left(r_{1}, r_{2}\right)}{z_{2}}\right)^{p_{2}} f\left(z_{1}, z_{2}\right)
$$

but as shown in [3] this is only true if

$$
\log a^{*} \leq \frac{a_{*}}{C\left(\log a_{*}\right)^{2}}
$$

where $a^{*}=\max \left(a_{1}, a_{2}\right), a_{*}=\min \left(a_{1}, a_{2}\right)$ and $C>0$ is a certain constant. Furthermore the condition is essentially sharp. (Note that in [3], $a_{1}$ and $a_{2}$ are replaced by the two central indices but these quantities are asymptotic.)

We can obtain (4.5) via (4.4) as we obtained (2.10) from (2.9) (albeit not as elegantly). By using Cauchy estimates one variable at a time we get uniform bounds on the error function in (4.4) much as we did in (3.12). Our error in estimating $\frac{\partial^{p_{1}+p_{2}}}{\partial z_{1}^{p_{1}} \partial z_{2}^{p_{2}}} f\left(z_{1}, z_{2}\right)$ is of the order

$$
o\left(\frac{\psi\left(a^{*}\left(r_{1}, r_{2}\right)^{p q / 4}\right)}{r_{1}^{p} r_{2}^{q}}\right) .
$$

This implies that (4.5) holds but with a much stronger condition than that obtained in [3], namely that

$$
a_{*} \geq K \sqrt{\psi\left(a^{*}\right)}
$$

for some $K>0$.

2. Fenton has shown in [3] that sets $E$ satisfying (4.3) occur naturally in two dimensional Wiman-Valiron theory and no smaller such set will suffice.

3. After the proof of Theorem 4.1 we will sketch how it can be extended to meromorphic functions in $\mathbf{C}^{2}$ with direct tracts.

\section{Proof of Theorem 4.1}

Let $\Phi:\left[t_{0}, \infty\right) \rightarrow \mathbf{R}^{+}$be convex and increasing in $r$. By Lemma 2.1 in [1] there is a set $E \subseteq \mathbf{R}^{+}$with meas $E \leq \kappa, \kappa>0$, such that

$$
\Phi(r+h) \leq \Phi(r)+\Phi^{\prime}(r) h+\epsilon(r)
$$

where $\epsilon(r) \rightarrow 0$ as $r \rightarrow \infty, r \notin E$, uniformly for

$$
|h| \leq \frac{1}{\sqrt{\psi\left(\Phi^{\prime}(r)\right)}} .
$$


What is not mentioned explicitly in the Lemma but is seen in its proof is that both $\kappa$ and $\epsilon(r)$ depend only on $\psi$ and not on $\Phi$. This is important in what follows.

Let $\Phi:\left[t_{0}, \infty\right) \times\left[t_{0}, \infty\right) \rightarrow \mathbf{R}^{+}$be convex as a function of two variables and increasing in each variable separately and set for $i=1,2$,

$$
\Phi_{i}\left(r_{1}, r_{2}\right)=\frac{\partial}{\partial r_{i}} \Phi\left(r_{1}, r_{2}\right)
$$

We obtain by (5.1)

$$
\Phi\left(r_{1}+h_{1}, r_{2}\right) \leq \Phi\left(r_{1}, r_{2}\right)+\Phi_{1}\left(r_{1}, r_{2}\right) h_{1}+\epsilon\left(r_{1}\right)
$$

for all $r_{1} \notin E_{r_{2}}$ and for all

$$
\left|h_{1}\right| \leq \frac{1}{\sqrt{\psi\left(\Phi_{1}\left(r_{1}, r_{2}\right)\right)}}
$$

where $\epsilon\left(r_{1}\right) \rightarrow 0$ as $r_{1} \rightarrow \infty$ uniformly in $r_{2}$. Since meas $E_{r_{2}} \leq \kappa$, we obtain

$$
\text { meas } E_{1} \cap\left[a, R_{1}\right] \times\left[a, R_{2}\right]=\int_{a}^{R_{2}} \int_{E_{r_{2}}} d r_{1} d r_{2} \leq \kappa R_{2}
$$

where $E_{1}=\cup_{r_{2}}\left(E_{r_{2}} \times\left\{r_{2}\right\}\right)$ We obtain a similar result for $\Phi\left(r_{1}, r_{2}+h_{2}\right)$ with a set $E_{2}$. Let $E=E_{1} \cup E_{2}$.

Lemma 5.1. Let $\Phi$ and $E$ be as above. Then for all $\left(r_{1}, r_{2}\right) \notin E$ and for $\left(h_{1}, h_{2}\right)$ in the rhombus $\Lambda\left(r_{1}, r_{2}\right)$ with vertices

$$
\begin{gathered}
\left( \pm 1 / \sqrt{\psi\left(\Phi_{1}\left(r_{1}, r_{2}\right)\right.}, 0\right) \quad \text { and }\left(0, \pm 1 / \sqrt{\psi\left(\Phi_{2}\left(r_{1}, r_{2}\right)\right.}\right) \\
\Phi\left(r_{1}+h_{1}, r_{2}+h_{2}\right) \leq \Phi\left(r_{1}, r_{2}\right)+\Phi_{1}\left(r_{1}, r_{2}\right) h_{1}+\Phi_{2}\left(r_{1}, r_{2}\right) h_{2}+o(1)
\end{gathered}
$$

uniformly as $r_{1} \rightarrow \infty$ and $r_{2} \rightarrow \infty$. The set $E$ satisfies

$$
\text { meas } E \cap\left[a, R_{1}\right] \times\left[a, R_{2}\right] \leq \kappa\left(R_{1}+R_{2}\right) \text {. }
$$

Proof. By the preceding discussion we obtain the inequalites

$$
\Phi\left(r_{1}+h_{1}, r_{2}\right) \leq \Phi\left(r_{1}, r_{2}\right)+\Phi_{1}\left(r_{1}, r_{2}\right) h_{1}+\epsilon\left(r_{1}\right)
$$

and

$$
\Phi\left(r_{1}, r_{2}+h_{2}\right) \leq \Phi\left(r_{1}, r_{2}\right)+\Phi_{2}\left(r_{1}, r_{2}\right) h_{2}+\epsilon\left(r_{2}\right)
$$

These inequalities measure the separation of the surface $z=\Phi\left(r_{1}, r_{2}\right)$ from its tangent plane at $\left(r_{1}, r_{2}\right)$ in the $r_{1}$ and $r_{2}$ directions respectively. Since $\Phi$ is convex the separation of the graph over the rhombus is largest at its vertices. The lemma is proved.

Let $\Lambda\left(r_{1}, r_{2}\right)$ be the rhombus in Lemma 5.1. By Lemma 5.1 and a logarithmic change of variables we obtain

Lemma 5.2. For all $\left(r_{1}, r_{2}\right) \notin E$, where E satisfies (4.3) and

$$
\left(\log \frac{s_{1}}{r_{1}}, \log \frac{s_{2}}{r_{2}}\right) \in \Lambda\left(r_{1}, r_{2}\right)
$$

we have

$$
\log M\left(s_{1}, s_{2}\right) \leq \log M\left(r_{1}, r_{2}\right)+a_{1}\left(r_{1}, r_{2}\right) \log \frac{s_{1}}{r_{1}}+a_{2}\left(r_{1}, r_{2}\right) \log \frac{s_{2}}{r_{2}}+o(1)
$$

uniformly as $r_{1} \rightarrow \infty$ and $r_{2} \rightarrow \infty$. 
Just as (3.2) gave us (3.8) in Theorem 2.1, (5.2) gives us a function

$$
u_{1}\left(z_{1}, z_{2}\right)=\log \left|f\left(z_{1}, z_{2}\right)\right|-\log M\left(r_{1}, r_{2}\right)-a_{1}\left(r_{1}, r_{2}\right) \log \frac{\left|z_{1}\right|}{r_{1}}-a_{2}\left(r_{1}, r_{2}\right) \log \frac{\left|z_{2}\right|}{r_{2}}
$$

subharmonic in each variable such that

$$
u_{1}\left(z_{1}, z_{2}\right)=o(1)
$$

uniformly in $\left.D\left(z_{1, r_{1}}, z_{2, r_{2}} ; r_{1} h_{1}, r_{2} h_{2}\right)\right)$ as $r_{1} \rightarrow \infty, r_{2} \rightarrow \infty$, where $\left(h_{1}, h_{2}\right) \in \Lambda\left(r_{1}, r_{2}\right)$ and $\left(r_{1}, r_{2}\right) \notin E$.

We note that the rectangle centered at the origin with side lengths $4 T_{1} / r_{1}$ and $4 T_{2} / r_{2}$ lies in $\Lambda\left(r_{1}, r_{2}\right)$, where $T_{1}$ and $T_{2}$ are given by (4.2). It follows that (5.3) holds uniformly in the closure of $D\left(z_{1, r_{1}}, z_{2, r_{2}} ; 2 T_{1}, 2 T_{2}\right)$.

We claim that $f$ is not zero in $D_{1}=D\left(z_{1, r_{1}}, z_{2, r_{2}} ; T_{1}, T_{2}\right)$. To see this, suppose that $f\left(\zeta_{1}, \zeta_{2}\right)=0$ for $\left(\zeta_{1}, \zeta_{2}\right) \in D_{1}$. Let $\zeta \in \mathbf{C}$ and consider

$$
w(\zeta)=f\left(z_{1, r_{1}}+\omega_{1} \zeta, z_{2, r_{2}}+\omega_{2} \zeta\right)
$$

where $\omega_{1}=\zeta_{1}-z_{1, r_{1}}$ and $\omega_{2}=\zeta_{2}-z_{2, r_{2}}$. Then $w$ is entire with $w(1)=0$. By definition $u_{1}-\log |f|$ is harmonic provided $z_{1}$ and $z_{2}$ are not 0 and so $u_{1}\left(z_{1, r_{1}}+\right.$ $\left.\omega_{1} \zeta, z_{2, r_{2}}+\omega_{1} \zeta\right)$ and $\log |w(\zeta)|$ have the same mass in $D_{1}$. Let $n(t)$ be the Riesz mass of $u_{1}\left(z_{1, r_{1}}+\omega_{1} \zeta, z_{2, r_{2}}+\omega_{1} \zeta\right)$ in $|\zeta| \leq t$. Note that $n(t) \geq 1$ for $t \geq 1$.

By Jensen's formula

$$
\begin{aligned}
\log 2 & \leq \int_{1}^{2} \frac{n(t)}{t} d t \leq \int_{0}^{2} \frac{n(t)}{t} d t \\
& =\frac{1}{2 \pi} \int_{0}^{2 \pi} u_{1}\left(z_{1, r_{1}}+2 \omega_{1} e^{i \theta}, z_{2, r_{2}}+2 \omega_{2} e^{i \theta}\right) d \theta-u_{1}\left(z_{1, r_{1}}, z_{2, r_{2}}\right)=o(1)
\end{aligned}
$$

as $\min \left(r_{1}, r_{2}\right) \rightarrow \infty$. The last equality follows by (5.3) and the fact that

$$
\left(z_{1, r_{1}}+2 \omega_{1} e^{i \theta}, z_{2, r_{2}}+2 \omega_{2} e^{i \theta}\right) \in \bar{D}_{1}
$$

for all $\theta$. This contradiction implies that $\log |f|$ is harmonic in $D_{1}$. Now we proceed exactly as in the proof of Theorem 2.1, modifying (3.10) to produce an analytic function $g_{1}$ in two variables whose real part is $u_{1}$. We use Borel-Carathéodory one variable at a time and obtain (4.4). The proof of Theorem 4.1 is complete.

To extend Theorem 4.1 to direct tracts we follow closely the arguments in [2]. For $a, b, c, d \in \mathbf{C}$ we define

$$
\mathcal{P}=\mathcal{P}(a, b, c, d)=\{(a+b z, c+d z): z \in \mathbf{C}\}
$$

to be a complex plane in $\mathbf{C}^{2}$. Let $\mathcal{D}$ be an unbounded open, connected set in $\mathbf{C}^{2}$ such that for any complex plane $\mathcal{P}$ the boundary of the set $\mathcal{D} \cap \mathcal{P}$ consists of piecewise smooth curves. If there is a function $f\left(z_{1}, z_{2}\right)$ which is analytic in $\mathcal{D}$, continuous in $\overline{\mathcal{D}}$, and such that $|f|>1$ in $\mathcal{D}$ while $|f|=1$ on $\partial D$, then we will call $\mathcal{D}$ a direct tract of $f$.

Define a subharmonic function $v$ by

$$
v\left(z_{1}, z_{2}\right)= \begin{cases}\log \left|f\left(z_{1}, z_{2}\right)\right| & \text { if }\left(z_{1}, z_{1}\right) \in \overline{\mathcal{D}} \\ 0 & \text { otherwise }\end{cases}
$$

We denote by $B\left(r_{1}, r_{2}\right)$ the maximum of $v$ on the polydisc $D\left(0,0 ; r_{1}, r_{2}\right)$ with $a_{1}$ and $a_{2}$ the partial derivatives of $B$ with respect to $\log r_{1}$ and $\log r_{2}$. Given $r_{1}, r_{2}>0$, let $\left(z_{1, r_{1}}, z_{2, r_{2}}\right)$ be as in Theorem 4.1 with $\log \left|f\left(z_{1, r_{1}}, z_{2, r_{2}}\right)\right|=B\left(r_{1}, r_{2}\right)$ and $\left|z_{1, r_{1}}\right|=r_{1}$, $\left|z_{2, r_{2}}\right|=r_{2}$. Our goal is to show that (4.4) still holds in $D\left(z_{1, r_{1}}, z_{2, r_{2}} ; T_{1}\left(r_{1}, r_{2}\right), T_{2}\left(r_{1}\right.\right.$, 
$\left.r_{2}\right)$ ), where $T_{1}\left(r_{1}, r_{2}\right)$ and $T_{2}\left(r_{1}, r_{2}\right)$ are given by (4.2). By a rescaling (to fit the notation in [2]) our methods in Theorem 4.1 give a subharmonic function

$$
u_{1}\left(z_{1}, z_{2}\right)=v\left(z_{1}, z_{2}\right)-B\left(r_{1}, r_{2}\right)-a_{1}\left(r_{1}, r_{2}\right) \log \frac{\left|z_{1}\right|}{r_{1}}-a_{2}\left(r_{1}, r_{2}\right) \log \frac{\left|z_{2}\right|}{r_{2}}
$$

such that (5.3) holds in $D\left(z_{1, r_{1}}, z_{2, r_{2}} ; 512 T_{1}\left(r_{1}, r_{2}\right), 512 T_{2}\left(r_{1}, r_{2}\right)\right)$. As in the proof of Theorem 4.1 we are done once we prove that $v$ is harmonic in $D_{1}=D\left(z_{1, r_{1}}, z_{2, r_{2}} ; T_{1}\left(r_{1}\right.\right.$, $\left.\left.r_{2}\right), T_{2}\left(r_{1}, r_{2}\right)\right)$. By definition of $v$ this will be accomplished if we can show that $D_{1} \subseteq \mathcal{D}$ for $r_{1}$ and $r_{2}$ sufficiently large.

So we assume that

$$
\left(\xi_{1}, \xi_{2}\right) \in D_{1} \backslash \mathcal{D}
$$

and further that $\left(\xi_{1}, \xi_{2}\right)$ is closest to $\left(z_{1, r_{1}}, z_{2, r_{2}}\right)$; this ensures that the line segment from $\left(z_{1, r_{1}}, z_{2, r_{2}}\right)$ to $\left(\xi_{1}, \xi_{2}\right)$ lies in $\mathcal{D}$.

Let $\mathcal{D}\left(r_{1}, r_{2}\right)$ be the component of $\mathcal{D} \cap \mathcal{P}$ containing $\left(z_{1, r_{1}}, z_{2, r_{2}}\right)$, where $\mathcal{P}=$ $\mathcal{P}\left(z_{1, r_{1}}, \xi_{1}-z_{1, r_{1}}, z_{2, r_{2}}, \xi_{2}-z_{2, r_{2}}\right)$. We note that $f$ restricted to $\mathcal{P}$ can be considered an analytic function of one variable which is large at the point $\left(z_{1, r_{1}}, z_{2, r_{2}}\right) \in \mathcal{D}\left(r_{1}, r_{2}\right)$ and whose modulus is no greater than 1 on $\partial \mathcal{D}\left(r_{1}, r_{2}\right)$. Thus $\mathcal{D}\left(r_{1}, r_{2}\right)$ is an unbounded connected set in $\mathcal{P}$ and can be thought of as a direct tract for the restricted function. Furthermore (5.3) holds when restricted to $\mathcal{P}$. Thus we can follow the one variable methods in [2] to show that $D_{1} \cap \mathcal{P} \subseteq D\left(r_{1}, r_{2}\right)$. This implies that $\left(\xi_{1}, \xi_{2}\right) \in \mathcal{D}\left(r_{1}, r_{2}\right)$ and hence in $D_{1}$, a contradiction.

\section{Functions of finite order: density results}

Let $f$ be meromorphic in $\mathbf{C}$ with a direct tract $D$ and let $u$ be the associated subharmonic function which equals $\log |f|$ on $D$ and 0 elsewhere and let $T(r)$ be as in (2.6). In ([1], Theorems 1.1 and 1.2) it is not only shown that outside a set of $r$ of finite logarithmic measure

$$
f(z)=(1+o(1)) f\left(z_{r}\right)\left(\frac{z}{z_{r}}\right)^{a(r, u)}
$$

for $z \in \Delta\left(z_{r}, T(r)\right)$, but also an example is given showing that the magnitude of $T(r)$ is best possible. The example that establishes this is of infinite order. For finite order we can do better.

Theorem 6.1. Let $f$ be meromorphic of finite order $\lambda$ with a direct tract $D$. Then for every function $T(r)$ satisfying $T(r)=o(r / \sqrt{a(r, u)})$, there exists a set $E$ of (upper) logarithmic density 0 such that (6.1) holds for $z \in \Delta\left(z_{r}, T(r)\right)$, as $r \rightarrow \infty$, $r \notin E$.

We remind the reader that the upper logarithmic density of a set $E \subseteq \mathbf{R}^{+}$is defined by

$$
\overline{\operatorname{logdens}} E=\limsup _{r \rightarrow \infty} \frac{\operatorname{logmeas}(E \cap[1, r])}{\log r} .
$$

Similarly the lower logarithmic density, $\underline{\log d e n s} E$ is defined using liminf. When logdens $E=\overline{\operatorname{logdens}} E=L$, we say that the logarithmic density of $E$ is $L$ and write $\overline{\operatorname{logdens}} E=L$.

Theorem 6.1 is true if $f$ is only assumed to have finite lower order. The exceptional set would then have lower logarithmic density 0 . The generalization to lower order is standard and we omit it. 
We note that it is sufficient (the argument is standard) to show that, given $\epsilon>0$ the conclusion of Theorem 6.1 holds outside a set of upper logarithmic density less that $\epsilon$.

Our goal is to improve (3.2) so that $T(r)$ is at least $o(r / \sqrt{a(r, u)})$ except on a set of logarithmic density 0. Theorem 6.1 follows from this by the argument in Section 12 of [2]. To do this we need a slight variant due to Fenton [5, Theorem 1] of a version of the Borel-Nevanlinna lemma proved by Bergweiler [1, Lemma 2.1].

Let $S:[a, \infty) \rightarrow \mathbf{R}^{+}$be an increasing function such that

$$
\limsup _{r \rightarrow \infty} \frac{\log S(r)}{r} \leq \lambda<\infty
$$

Fix $\epsilon>0$. Let $g(t)$ be a nonnegative function bounded above and below on compact subsets of $\mathbf{R}^{+}$such that

$$
g(r)=o(r)
$$

as $r \rightarrow \infty$ and let

$$
h(t)=\frac{1}{\lambda_{1} t}
$$

where $\lambda / \lambda_{1}<\epsilon$. Then the proof of Theorem 1 in [5] shows that with $S=S(r)$

$$
S(r+g(S) h(S)) \leq S+g(S)
$$

and

$$
S(r-g(S) h(S)) \geq S-g(S)
$$

except on a set $E_{\epsilon}$ of $r$ contained in the union of intervals of the form $\left[r_{n}, r_{n}+\right.$ $\left.g\left(S_{n}\right) h\left(S_{n}\right)\right]$ where $S_{n}=S\left(r_{n}\right)$ and $r_{n+1} \geq r_{n}+g\left(S_{n}\right) h\left(S_{n}\right)$. Furthermore

$$
g\left(S_{n}\right) h\left(S_{n}\right) \leq(1+o(1)) \int_{S_{n}}^{S_{n}+g\left(S_{n}\right)} h(t) d t .
$$

(We remark that in [5] a more general $h$ gives only a constant $K$ in (6.7) rather than $1+o(1)$.)

Let $r \geq a$ be arbitrary and let $n$ be the largest positive integer such that $r_{n}+$ $g\left(S_{n}\right) h\left(S_{n}\right) \leq r$. Then by (6.7) and (6.3)

$$
g\left(S_{n+1}\right) h\left(S_{n+1}\right) \leq(1+o(1)) \log \left(1+g\left(S_{n+1}\right) / S_{n+1}\right)=o(1) .
$$

Now

$$
\operatorname{meas}\left(E_{\epsilon} \cap(1, r)\right) \leq \sum_{j=1}^{n+1} g\left(S_{j}\right) h\left(S_{j}\right) .
$$

Then by (6.7) and (6.8) we obtain

$$
\operatorname{meas}\left(E_{\epsilon} \cap(1, r)\right) \leq(1+o(1)) \int_{r_{1}}^{S_{n}+g\left(S_{n}\right)} h(t) d t .
$$

By (6.3), the definition of $h$ and (6.2) we obtain

$$
\operatorname{meas}\left(E_{\epsilon} \cap(1, r)\right) \leq \frac{\lambda+o(1)}{\lambda_{1}} \log r
$$

and it follows that $E_{\epsilon}$ has upper logarithmic density at most $\epsilon$. 
Let $a(r)$ be as in Theorem 6.1. Since $f$ has order $\lambda, u$ and hence $a(r, u)=a(r)$ have orders at most $\lambda$, we apply the argument above to the function $T(x)=a\left(e^{x}\right)=$ $a(r)$. We obtain from (6.5) and (6.6) that for all $r \notin E_{\epsilon}$

$$
a\left(r e^{g(a(r)) / \lambda_{1} a(r)}\right) \leq a(r)+g(a(r))
$$

and

$$
a\left(r e^{-g(a(r)) / \lambda_{1} a(r)}\right) \geq a(r)-g(a(r)) .
$$

Since $a(r)=d B(r) / d \log r$ we have

or

$$
B(s)=B(r)+\int_{r}^{s} a(t) d t
$$

$$
B(s)=B(r)-\int_{s}^{r} a(t) d t
$$

for $s \geq r$ or $s \leq r$ respectively. Using (6.10) and (6.11), if $\left|\log \frac{s}{r}\right| \leq g(a(r)) / \lambda_{1} a(r)$ we obtain for all $r \notin E_{\epsilon}$

$$
B(s) \leq B(r)+a(r) \log \frac{s}{r}+g(a(r))\left|\log \frac{s}{r}\right| .
$$

We obtain (3.2) if also $g(a(r))\left|\log \frac{s}{r}\right|=o(1)$. Both of these requirements are met if $g(t)=o(\sqrt{t})$. Such a choice of $g$ implies that (3.2) holds for all $s$ such that $\left|\log \frac{s}{r}\right|$ is less than any given function that is $o(1 / \sqrt{a(r)})$, and is always possible if $T(r)=o(r / \sqrt{a(r)}$. As mentioned above this is enough to prove Theorem 6.1.

\section{Functions of finite order: Pólya Peaks}

An increasing, positive sequence $r_{n} \rightarrow \infty$ is called a sequence of Pólya Peaks of order $\sigma$ of the function $k(r)$ if there exist positive sequences $A_{n} \rightarrow \infty$ and $\epsilon_{n} \rightarrow 0$ such that

$$
\frac{k(r)}{k\left(r_{n}\right)} \leq\left(1+\epsilon_{n}\right)\left(\frac{r}{r_{n}}\right)^{\sigma} \quad\left(A_{n}^{-1} r_{n} \leq r \leq A_{n} r_{n}\right)
$$

It is known that if $k$ is also continuous and $\mu<\infty$ and $\lambda$ are the lower order and order respectively of $k$ then $k(r)$ has a sequence of Pólya Peaks of order $\sigma$ for every $\sigma \in[\mu, \lambda]$ if $\lambda<\infty$ and for every $\sigma \in[\mu, \infty)$ otherwise. In the proof of this result (see [10, p. 710]) what is actually proved is that given any positive sequence $\eta_{n} \rightarrow 0$,

$$
\frac{k(r)}{k\left(r_{n}\right)} \leq\left(\frac{r}{r_{n}}\right)^{\sigma}\left(\frac{r_{n}}{r}\right)^{\eta_{n}} \quad\left(A_{n}^{-1} r_{n} \leq r \leq r_{n}\right)
$$

and

$$
\frac{k(r)}{k\left(r_{n}\right)} \leq\left(\frac{r}{r_{n}}\right)^{\sigma}\left(\frac{r}{r_{n}}\right)^{\eta_{n}} \quad\left(r_{n} \leq r \leq A_{n} r_{n}\right) .
$$

The two inequalities are combined to form (7.1) by setting $\epsilon_{n}=A_{n}^{\eta_{n}}-1$. If in (7.1) there is no apriori mention of the relationship between $\epsilon_{n}$ and $\eta_{n}$, then (7.1) is a much weaker requirement than (7.2) and (7.3) when $r$ and $r_{n}$ are asymptotic, which is exactly the case in which we are interested. (We remark that we only use (7.3) in what follows.)

Functions of finite lower order behave fairly regularly on a sequence of Pólya Peaks and many of the inequalities involving functionals of Nevanlinna theory are proved at the Pólya Peaks of one of these functionals (see [10, Sections 9.6 and 9.7]). We show that on a sequence $s_{n}$ asymptotically close to a sequence of Pólya 
Peaks, the inequality (3.2) holds with $T\left(s_{n}\right)$ at least as large as $o\left(s_{n} / \sqrt{a\left(s_{n}\right)}\right)$ for any subharmonic function $u$ of finite lower order. Thus as in Theorem 6.1 if $u=\log |f|$ on $D$ and 0 elsewhere we obtain Wiman-Valiron disks of radii at least $o\left(s_{n} / \sqrt{a\left(s_{n}\right)}\right)$ for $f$.

Let $u$ be subharmonic of finite lower order $\mu$. Fix $h_{n}=h \geq 0$. Define $s_{n}=r_{n} e^{h}$, $t_{n}=s_{n} e^{h}=r_{n} e^{2 h}$. Then by (7.3) applied to $a(r)$ we obtain as $h \rightarrow 0$

$$
\begin{aligned}
B\left(t_{n}\right) & =B\left(s_{n}\right)+\int_{s_{n}}^{t_{n}} a(t) \frac{d t}{t} \leq B\left(s_{n}\right)+a\left(t_{n}\right) h \leq B\left(s_{n}\right)+a\left(r_{n}\right) e^{\left(\sigma+\eta_{n}\right) 2 h} h \\
& \leq B\left(s_{n}\right)+a\left(s_{n}\right) e^{\left(\sigma+\eta_{n}\right) 2 h} h=B\left(s_{n}\right)+a\left(s_{n}\right) h+a\left(s_{n}\right)\left(e^{\left(\sigma+\eta_{n}\right) 2 h}-1\right) h \\
& =B\left(s_{n}\right)+a\left(s_{n}\right) h+a\left(s_{n}\right)(1+o(1)) 2 \sigma h^{2} .
\end{aligned}
$$

Similarly by (7.3) we obtain as $h \rightarrow 0$

$$
\begin{aligned}
B\left(r_{n}\right) & =B\left(s_{n}\right)-\int_{r_{n}}^{s_{n}} a(t) \frac{d t}{t} \leq B\left(s_{n}\right)-a\left(r_{n}\right) h \leq B\left(s_{n}\right)-a\left(s_{n}\right) e^{-\left(\sigma+\eta_{n}\right) h} h \\
& =B\left(s_{n}\right)+a\left(s_{n}\right)(-h)+a\left(s_{n}\right)\left(e^{-\left(\sigma+\eta_{n}\right) h}-1\right)(-h) \\
& =B\left(s_{n}\right)+a\left(s_{n}\right)(-h)+a\left(s_{n}\right)(1+o(1)) \sigma h^{2} .
\end{aligned}
$$

We have proved that so long as $r_{n} e^{h}=s_{n}$ and $|h|=o\left(1 / \sqrt{a\left(s_{n}\right)}\right)$ then

$$
B\left(s_{n} e^{h}\right) \leq B\left(s_{n}\right)+a\left(s_{n}\right) h+o(1)
$$

and

$$
B\left(s_{n} e^{-h}\right) \leq B\left(s_{n}\right)+a\left(s_{n}\right)(-h)+o(1) .
$$

By (7.4) and (7.5) the inequality

$$
B\left(s_{n} e^{t}\right) \leq B\left(s_{n}\right)+a\left(s_{n}\right) t+o(1)
$$

holds at the endpoints of the interval $[-h, h]$. By the convexity of $B,(7.6)$ holds for all $t \in[-h, h]$ where the $o(1)$ approaches 0 uniformly in this interval and depends only on $\sigma$. It follows by the method in [2] that Wiman-Valiron disks around $s_{n}$ have radii greater than $s_{n} \rho\left(s_{n}\right)$ where $\rho\left(s_{n}\right)=o\left(1 / \sqrt{a\left(s_{n}\right)}\right)$.

\section{References}

[1] Bergweiler, W.: The size of Wiman-Valiron discs. - Complex Var. Elliptic Equ. 56, 2011, $13-33$.

[2] Bergweiler, W., P. J. Rippon, and G. M. Stallard: Dynamics of meromorphic functions with direct or logarithmic tracts. - Proc. London Math. Soc. 97, 2008, 369-400.

[3] Fenton, P. C.: Wiman-Valiron theory in two variables. - Trans. Amer. Math. Soc. 347, 1995, 4403-4412.

[4] Fenton, P. C.: Polynomial approximation of entire function of two variables. - Complex Var. Theory Appl. 40, 2000, 211-230.

[5] Fenton, P. C.: On a lemma of Bergweiler. - Contemp. Math. (to appear).

[6] Fenton, P. C., and J. Rossi: ODEs and Wiman-Valiron theory in the unit disc. - J. Math. Anal. Appl. 367, 2010, 137-145.

[7] Fenton, P. C., and J. Rossi: Two variable Wiman-Valiron theory and PDEs. - Ann. Acad. Sci. Fenn. Math. 35, 2010, 571-580. Errata ArXiv:1009.6225.

[8] Fenton, P. C., and J. Rossi: Subharmonic functions that are harmonic when they are large. - Anal. Math. Phys. 2014, 115-130. 
[9] Hayman, W. K.: The local growth of power series: a survey of the Wiman-Valiron method. - Canad. Math. Bull. 17, 1974, 317-358.

[10] Hayman, W. K.: Subharmonic functions. Volume 2. - London Math. Soc. Monogr. Ser. 20, 1990.

[11] Langley, J. K., and J. Rossi: Wiman-Valiron theory for a class of meromorphic functions in the unit disc. - Proc. Royal Irish Math. Soc. 2, 2014, 137-148.

Received 1 May 2015 • Accepted 25 September 2015 